\title{
Británicos en la sociedad de Buenos Aires (1804-1810)
}

\author{
British in the Buenos Aires society (1804-1810)
}

Roberto Dante Flores*

RESUMEN

Las dos invasiones inglesas al Río de la Plata $(1806 / 7)$ trajeron consecuencias de diversa índole: política, económica y demográfica. Vemos que constituyeron un quiebre, una especie de bisagra histórica entre dos momentos. Contribuyeron a la transición de una sociedad colonial -regulada por España- a otra independiente en lo político, pero dependiente en lo comercial de Gran Bretaña. Y, demográficamente, más cosmopolita. Sin embargo, estos tres aspectos están íntimamente vinculados y nos parece necesario realizar un trabajo donde aparezcan en forma conjunta, para mostrar una realidad social más completa. Nuestro artículo tomará como eje histórico las invasiones inglesas al Río de la Plata y la matriz de datos será los padrones de la población de Buenos Aires durante 1804, 1806/ 7y 1810. Durante nuestra investigación buscaremos responder a los siguientes interrogantes: 1) ¿Con qué fin vinieron los británicos a Buenos Aires? 2) ¿Qué actividades realizaron? 3) ¿Cuáles fueron sus vínculos con la sociedad porteña?

PALAVRAS-CHAVE: Británicos -Buenos Aires; Población - Sociedad

\section{ABSTRACT}

The two British invasions of the Río de la Plata (1806/7) brought consequences of various kinds: political, economic and demographic. We see that constituted to a break, a kind of hinge between two historical moments. They contributed to the transition from a social colonial regime, regulated by Spain, to another one politically independent, but, commercial-dependent from Great Britain and more demographically cosmopolitan. However, these three aspects are closely linked and, therefore, it seems necessary to work in a piece, in which they appear linked to one another, so as to show a complete social reality. The British invasions at the Rio de la Plata are axis from this work, and the census of the population of Buenos Aires during 1804, 1806 / 7 and 1810 are the data matrix. Our research will seek to answer the following questions:1) Why did the British finally come to Buenos Aires? 2) What activities did they conduct? 3) What were their links with the Buenos Aires society?

KEY-WORDS: British - Buenos Aires; Population- Society

\section{Introducción}

En el inicio del siglo XIX, el Virreinato del Río de la Plata estaba regido por leyes de la corona española que prohibían el comercio con Inglaterra. En Europa, el amenazante emperador francés Napoleón I (1804) empezaba a controlar el continente, y tenía como objetivo derrotar a los británicos desembarcando sus tropas en Inglaterra. Sin embargo, sus planes de invasión fueron abortados en la Batalla de Trafalgar (1805) donde fue derrotada la flota conjunta franco-española. Entonces, en lugar de la estrategia militar, Napoleón optó por el bloqueo comercial a las islas británicas, prohibiendo a Francia y sus aliados (entre ellos España) el comercio de productos

\footnotetext{
* Universidad de Buenos Aires - Argentina. Dirección electrónica: rodanteflores@gmail.com
} 
británicos. Esta medida no afectaba formalmente al comercio en el Río de la Plata porque esa prohibición ya existía. A pesar de la misma, los ingleses en Buenos Aires con el consentimiento del gobierno británico y la complicidad virreinalcomercializaban ilegalmente productos en barcos neutrales (Street, Graham-Yooll) La alianza entre Francia y España alertó a los británicos sobre la posibilidad de que los franceses afectaran ese lucrativo contrabando suramericano, incluso temían una invasión francesa a las colonias suramericanas. En ese contexto político, y alentados por un ex ministro de los Estados Unidos, Home Popham y William Beresford decidieron adelantarse a Napoleón Bonaparte y tomaron Buenos Aires (Street, Alzaga)

Las dos invasiones inglesas al Río de la Plata (1806/7) trajeron consecuencias de diversa índole: política, económica y demográfica. Vemos que constituyeron un quiebre, una especie de bisagra histórica entre dos momentos. Contribuyeron a la transición de una sociedad colonial -regulada por España- a otra independiente en lo político, pero dependiente en lo comercial, de Gran Bretaña. Y, demográficamente, más cosmopolita. Sin embargo, estos tres aspectos están íntimamente vinculados y nos parece necesario realizar un trabajo donde aparezcan en forma conjunta, para mostrar una realidad social más completa.

Luego de la primera invasión británica a Buenos Aires (27 de junio de 1806) vino la resistencia porteña, la derrota y prisión del general Beresford junto a sus soldados (12 de agosto de 1806). Con desconocimiento de esa derrota, “a fines de 1806, unos 2000 comerciantes y aventureros británicos alentados por la noticia de que Beresford había capturado Buenos Aires, partieron rumbo al Río de la Plata acompañando a la expedición de refuerzo de Samuel Auchmuty". ${ }^{2}$ Pero, al inicio de febrero de 1807, la escuadra británica que escoltó a los comerciantes, tomó Montevideo y esperó la llegada del general Whitelocke, con miras al ataque de Buenos Aires. Luego del ataque inglés a la capital del virreinato, y de la exitosa defensa de la ciudad ( 7 de julio de 1807), muchos comerciantes británicos volvieron a Inglaterra o a Brasil. Sin embargo, algunos regresaron a Buenos Aires a comercializar y se radicaron en la ciudad. Más adelante (1809), constituyeron el Comité de Comerciantes Británicos, primera institución británica en Buenos Aires. Ellos representaban a casas de comercio familiares con sede en Liverpool, Glasgowy Londres. (HANON, 2005).

\footnotetext{
2 Hanon (2005, p 22). En la segunda invasión británica de 1807 “El trabajo mayor para afianzar el gobierno inglés fue hecho por los comerciantes, de los cuales alrededor de 2000 vinieron (a Montevideo) en 70 barcos mercantes que entraron con los conquistadores y en los otros que llegaron más tarde", Street (1967, p 79). Las listas de los barcos comerciantes británicos arribados a Montevideo se publicaron en Southern Star, periódico inglés editado en Montevideo entre el 30 de mayo y el 4 de julio de 1807.
} 
Estas afirmaciones han despertado nuestro interés en conocer si los ingleses incrementaron su número entre la población porteña, en qué porcentaje, y a qué se debió. Entonces, el problema planteado es: ¿En que medida influyeron las invasiones inglesas en los cambios de esa población?

\section{Hipótesis}

Las invasiones inglesas a Buenos Aires (1806/7) favorecieron el incremento de la población porteña de origen británico, y ésta se orientó principalmente a la actividad comercial.

\section{Método}

Nuestra monografía tomará como eje histórico las invasiones inglesas al Río de la Plata y la matriz de datos será los padrones de la población de Buenos Aires durante 1804, 1806/ 7 y 1810. Durante nuestra investigación buscaremos responder a los siguientes interrogantes:

1) ¿Con qué fin vinieron los británicos a Buenos Aires?

2) ¿Qué actividades realizaron?

3) ¿Cuáles fueron sus vínculos con la sociedad porteña?

En primer lugar, para lograr el objetivo de dar respuestas adecuadas a la realidad, volcaremos sobre planillas los datos de la población según su origen y condición. Luego, realizaremos comparaciones de los mismos, antes y después de las invasiones. Por último, apoyándonos en otros documentos y cartas citadas por autores, intentaremos sacar conclusiones que corroboren o desmientan nuestra hipótesis.

\section{Objeto de la Investigación}

En un plano general de introducción describiremos -en base a fuentes secundarias indirectas- las líneas principales del contexto político de las potencias europeas y su relación con el Virreinato. Con la luz de esa información buscaremos comprender el sentido de las resoluciones dictadas -previas a la revolución de Mayosobre el comercio y la residencia de británicos en Buenos Aires.

Finalmente, el nivel más profundo de la investigación será social-demográfico. Este nos permitirá - en base a fuentes primarias- señalar si se dieron cambios en la población de Buenos Aires al interactuar los británicos con la sociedad porteña establecida. 


\section{Características de la Población de Buenos Aires}

Aproximarse al conocimiento de la población de Buenos Aires en el período previo a la revolución de Mayo de 1810 requiere la confrontación de algunas fuentes documentales. En este caso serán el relato de un observador inglés, algunas cartas y, aunque incompletos, los padrones de la época.

La capital del virreinato del Río de la Plata estuvo abierta desde épocas tempranas a la residencia de extranjeros pese a repetidas órdenes de expulsión que nunca se concretaban Por ejemplo Jaime Badal en 1804 figura en una lista del gobierno con orden de expulsión, causa: religión protestante. En marzo de 1806 una autoridad católica solicita al gobierno que se revoque la orden, causa: estar instruyéndose en la religión católica. En 1810 Badal continuaba en Buenos Aires, se había casado con J osefina Morales y tenían tres hijos.

Una pragmática de Carlos IV distinguía:

Avecindado: Extranjeros que debían jurar fidelidad a la religión católica y a la soberanía real renunciando al fuero de extranjería y a la protección de su país de origen.

Transeúnte: Extranjeros que obtenían licencia para una permanencia temporaria y les estaba prohibido ejercer artes liberales u oficios mecánicos sin avecindarse.

De la normativa surge que la conditio sine qua non para adquirir carácter de ciudadano de Buenos Aires era ser católico. Esta razón fue el principal motivo para realizar el empadronamiento del año 1804. Las causas fueron expuestas por el virrey de Sobremonte mediante un Bando Real del 10 de Octubre del mismo año. El objetivo era reconocer los extranjeros residentes en la ciudad de Buenos Aires con el fin de darles la condición de avecindados, transeúntes, o bien intimarlos a ser embarcados. Así comienzan a relatar los Alcaldes de los distintos cuarteles de la Ciudad de Buenos Aires sus respectivos informes de los que obtuvimos la información sobre los extranjeros de la Ciudad.

Relación de los extrangeros que en Observancia del Bando Real mandado publicar el 10 de Octubre proximo pasado por el Ex.mo S.or Marques de Sobremonte, Virrey y Capitan Gral. de estas Provincias del Rio de la Plata el 10 de octubre último se han presentado a su respectivo Alcalde

En algunos casos los alcaldes eran más precisos y extensos en la redacción del informe, lo cual nos permite conocer en detalle las causas del empadronamiento: 
En cumplimiento de lo mandado por V. E. en oficio de 10 de octubre ult.. que relativo al Vando R.l publicado en dho dia sobre los extranjeros resid. En est Capital, se digno comunicar al Alc. Informante, dirige este a las Superior. Manos de V.E. la adjunta Relación de los q. havitan en el Quartel Na 14 de syu cargo y se han presentado bajo el termino del mes prefijado en el indicado Vando, con distinc. de la Religión que profesan, del lugar de su origen, su estado, ocupación, oficio o ejercicio, y de los bienes raíces y muebles q. poseen: Quedando igualmente el Alc con el cuidado de indagar los demas extranjeros del mismo Quartel q no se huviesen presentado, para comunicarselo a V. E. oportunamente, y dar de este modo el mas perfecto lleno asu citado su. Oficio

Dios Nro S. Gue a V. E. m. a. Buenos Ayres 10 de noviembre de 1804.

Exmo Sr J osef Nadal y Campos (RAVIGNANI , 1919, p. 158).

De la lectura del censo de extranjeros de 1804 surge que, aquellos que estaban dispuestos a convertirse al catolicismo quedaban a cargo de una autoridad religiosa. La misma era consultada al cabo de un tiempo para disponer la asignación de ciudadanía o su "extrañamiento". Así dice un documento rubricado por el virrey Sobremonte: “deverá pedirse informe al Prelado local del convento de San Francisco sobre el particular de su reconciliación que expresan la citada relación en esta parte para disponer en su vista lo conveniente". (RAVIGNANI , 1919, p. 191-192). La reconciliación con la Iglesia era una práctica relativamente frecuente entre los extranjeros que se radicaban en Buenos Aires y contraían matrimonio. En 1804 había un total de 28 británicos con la siguiente proporcionalidad, según su estado civil:

\section{Solteros $9(32,14 \%)$ \\ Casados $8(28,57 \%)$ \\ Sin Datos $11(39,28 \%)$}

Estos últimos son aquellos que no tienen registrado su estado civil. De los casados hay dos que figuran "casado con inglesa" y un tercero -Jorge Aliburcon"casado con Martina Vrit", apellido que nos parece - con alguna deformación- de origen británico. Además J aime Vidal es un extranjero registrado como "islandés, católico, casado con María Francisca Ridam, inglesa católica”. La palabra islandés puede ser un error de escritura y debería leerse irlandés, considerando que difícilmente hubiera arribado a Buenos Aires un islandés católico casado con una inglesa, también católica. Por lo tanto, atendiendo a estos posibles ciudadanos de origen británico deberíamos ampliar el número de ellos a 32. Sin embargo, preferimos mantener el número 28 porque es el que determina el censo, ya que las esposas no figuran con todos sus datos. En el cuartel número 4 se registra una mujer inglesa, Maria de los Angeles Garate de 
Elezmar, casada con J osé Castellano. No tenemos más datos, pero llama a la atención éstos y otros nombres españoles entre los ingleses censados. Quizá se deba a que fueron recibidos en la Iglesia católica y cambiaron sus nombres adoptando otros de origen español. De todos modos hay un caso de matrimonio de nacionalidad mixta, David Reid, médico católico, escocés, casado con española. La mayoría de los matrimonios, según este censo, se daba entre británicos. ¿Porqué no había más casamientos entre británicos y españolas? Gillespie afirma que había una barrera.

La única barrera para vínculos más estrechos era la diferencia de credo, que con sólo sacrificarlo, las damas hubieran considerado el rango militar de sus admiradores como consideración de menor importancia. Una de ellas se casó con un cadete del cuerpo de Santa Helena, que se había convertido voluntariamente y poco después recibió una comisión de capitán del ejército de Buenos Aires. Mucha elocuencia sacerdotal se gastó en esta santa lucha. (GILLESPIE , 1994, p. 82).

Esta observación de primera fuente nos indica que los matrimonios mixtos tenían como condición la conversión del inglés a la religión católica. Tarea trabajosa, según expresa el autor inglés. En resumen, de ocho británicos casados (seis y dos viudos) sólo de uno tenemos certeza que se casó con una persona española. Sin embargo, la inglesa Maria Isabel de los Angeles Garate de Elezmar casada con J osé Castellano -probablemente español- también podría ser incluida dentro de los matrimonios de nacionalidad mixta. Estos dos matrimonios mixtos representan el 7\% de la población británica censada. Los británicos casados con miembros de la misma comunidad, con certeza, son cuatro, es decir el 14\% del total de la población británica censada en 1804.

El empadronamiento de extranjeros de 1807 tuvo motivaciones diferentes al de 1804. Para señalar las razones de su realización transcribimos, a modo de ejemplo, la lista del cuartel número 8. La autoridad del lugar registró una serie de nombres encabezada con la siguiente leyenda: "Razón de los Estrangeros residentes en el Cuartel Num. 8". Luego de los nombres (34 individuos), figura "nación”, "edad", "estado", "ocupación", "tipo de residencia” y "cuerpo en que sirve”, al pie de página constan fecha y firma del alcalde: "Buenos Ayres, 28 de Febrero de 1807. Antonio Piran". (RAVIGNANI , 1919, p. 158). Algunos resúmenes de cuarteles están fechados en 1806 y el cuartel nro 1 encabeza con la inscripción "padrones practicados en los años 1806 y 1807". Los mismos fueron realizados entre fines de 1806 y principios de 1807.

$\mathrm{Si}$ comparamos los empadronamientos surgen evidentes diferencias causales que motivaron su realización. En 1804 se pone énfasis en la "religión" profesada por el 
extranjero, y en sus "bienes". En 1807 estos factores desaparecen del cuestionario censal y aparecen la "edad" y "el cuerpo en que sirve". En otros términos, la primera invasión inglesa de 1806 hizo cambiar el eje de los censos de extranjeros residentes, privilegiando las razones de índole estratégico-militar.

Buenos Aires a principios del siglo XVIII era una ciudad relativamente pequeña. Durante la primera invasión inglesa la población no excedía de 41mil habitantes. Este dato lo obtenemos del capitán Alexander Gillespie, observador minucioso e informante británico de los aspectos sociales, políticos y económicos de Buenos Aires, y de las provincias del Plata. Cuando se intenta confirmar este dato a la luz del padrón de 1807 vemos que la cifra es aproximadamente la mitad (21.313). Aunque, considerando que sólo tenemos el registro de 10 cuarteles en forma completa, y faltarían otros 10 (hay datos fragmentarios hasta el cuartel 20), la cifra dada por el autor británico puede ser tomada como muy cercana al número de habitantes porteños en ese momento. La distribución poblacional según el sexo es más difícil de precisar.

El vasto exceso de mujeres sobre la población masculina de Buenos Aires era saliente cuando fuimos dueños de la ciudad, y era igualmente notable que esta observación se invirtiese después de internarse un poco en el campo. Esto puede responder, en aquel tiempo, a las muchas emigraciones secretas de la ciudad, para reclutar sus ejércitos, que hasta entonces se habían compuesto de súbditos indios u otros estrechamente ligados a ellos por el color y las costumbres. (GILLESPIE, 1994, p. 86-87).

Para tratar de comprobar esta observación fuimos al empadronamiento de 1807, donde se registran criollos, españoles, indios, esclavos y extranjeros. En primer lugar nos encontramos con las siguientes dificultades metodológicas:

a) El padrón de febrero de 1807 tenía por objeto hacer un relevamiento de los hombres residentes en Buenos Aires con el fin de saber el número a disponer para la defensa, considerando el reciente ataque británico a Montevideo. ${ }^{3}$ Por lo tanto, no era interés primario del empadronador conocer el número de mujeres. Sólo registra a las mujeres en cuatro cuarteles (cuarteles 1, 2, 5y 6)

b) No sabemos si la observación de Gillespie es sobre el total de mujeres (incluyendo a las esclavas) o si se refiere únicamente a las mujeres libres, españolas y americanas. Talvez éste último sea el verdadero sentido de su afirmación.

${ }^{3}$ El 19 de enero de 1807 Sir Samuel Auchmuty avamzó sobre Montevideo. Roberts (2006, p 249). 
Pero, ante la inseguridad optamos por sumar a todas las mujeres (libres, españolas, americanas, pardas, indias y esclavas). El resultado de nuestro estudio sincrónico, en los cuatro cuarteles citados, muestra que: En febrero de 1807 el total de mujeres (5.139) representaban el 51,63 \% del total de la población (9.953). Sólo superaban en 1,63\% al total de hombres (Ver Cuadro 7). Este dato estaría indicando lo siguiente:

a) La observación de Gillespie posee cierta exageración.

b) Pocos hombres dejaron la ciudad con el objeto de prepararse para su reconquista.

La otra posibilidad es que Gillespie se refiera a las mujeres de la sociedad, excluyendo a las esclavas e indias. Aunque así fuera, igualmente el número de españolas y americanas no superaba, en demasía, al número de los españoles y americanos. La segunda afirmación de Gillespie, sobre las muchas emigraciones de hombres, nos llevó a realizar un estudio diacrónico de los cuarteles (1806/ 7-1810). Así determinaremos un nuevo porcentaje de mujeres respecto al de hombres, y su variación. Para ser precisos sólo comparamos los porcentajes de los mismos cuarteles. De 1806/ 7 únicamente poseemos registro de mujeres en cuatro cuarteles. El cuartel 1 de ese año posee 55,28\% de población femenina, pero no lo consideramos porque en 1810 no tenemos registro de dicho cuartel.

Cuadro 1- Población femenina cuartel

\begin{tabular}{|c|c|c|c|c|c|c|}
\hline \multirow[t]{2}{*}{ Cuartel } & \multirow{2}{*}{$\begin{array}{c}\text { Padrón } \\
\text { mujeres }\end{array}$} & \multicolumn{2}{|c|}{$1806 / 7$} & \multicolumn{3}{|c|}{\begin{tabular}{|l|} 
Padrón 1810 \\
\end{tabular}} \\
\hline & & total & $\%$ & mujeres & total & $\%$ \\
\hline cuartel 2 & 1219 & 2166 & 56,28 & 1241 & 2280 & 54,43 \\
\hline cuartel 5 & 2105 & 4331 & 48,6 & 1890 & 4237 & 44,61 \\
\hline cuartel 6 & 1475 & 2841 & 51,92 & 1248 & 2519 & 49,54 \\
\hline total & 4799 & 9338 & 51,39 & 4379 & 9036 & 48,46 \\
\hline
\end{tabular}

Entonces, en estos tres cuarteles, las mujeres de 1810 habían disminuido 2,93 \%, comparativamente al padrón de 1806/7. Pero, si sumamos el total de mujeres censadas en 1810 el porcentaje alcanza 46,45 \% (Ver Cuadro 8). Por lo tanto, la diferencia porcentual (51,39\%-46,45\%) del total de mujeres de ambos padrones, resultaría 5\% más bajo en 1810, respecto a 1806/7. Esto confirmaría la observación del autor británico. El retorno de los hombres a la ciudad, luego del éxodo secreto al interior para organizar la reconquista de la ciudad en 1806, provocó una caída porcentual del número de mujeres. 
Por otra parte, esa situación demográfica está en directa relación con la movilización de hombres en la revolución de Mayo de 1810: "Como la J unta Suprema se encontraba con el mando de todas las tropas que habían sido movilizadas, cuando se anunció la defección a su causa de Montevideo y Córdoba, estuvo capacitada para lanzar destacamentos contra las dos" (GILLESPIE, 1994, p. 243). El mando de todas las tropas se refiere a tropas constituidas por ciudadanos porteños enrolados voluntariamente en alguno de los regimientos existentes. Estos ciudadanos ya enrolados no figuran en el padrón realizado en 1810, éste sólo registra a los hombres en condiciones de ser incorporados a la milicia (los que "deben ser alistados") Así surge de las notas de los alcaldes:

Buenos Ayres, 2 de Abril de 1810.

Lista de los individuos que deven tomar las armas y alistarse en los cuerpos que gusten, desde edad de 18 a 45 años, en virtud de orden de Exmo. Sor. Virrey, y son los siguientes (nombres de ciudadanos). Firma Raimundo Real Cuartel no.19

Otro aspecto importante de la estructura demográfica de la ciudad son las características raciales de sus habitantes. Allí podemos observar cuáles fueron las relaciones que se formaron entre diferentes grupos étnicos, las relaciones de trabajo establecidas y sus variaciones con la llegada de los inmigrantes británicos.

En 1806 la población de Buenos Aires no excedía de 41 mil: la quinta parte era de blancos, siendo el resto una casta compuesta en variados estados de conexión y cambios progresivos, desde el negro hasta el tinte del europeo más rubio. (GILLESPIE, 1994, p. 53).

Para comprobar esta afirmación realizamos una detallada clasificación -según permiten los datos de los padrones- de la población según razas. Si consideramos blancos sólo a los españoles europeos el porcentaje es del 42 \% de la población de 1807, casi 1/2 y no 1/5 según Gillespie. La proporción de españoles desciende 34\% en 1810, llegando a 7,9\% de la población total. En el padrón de febrero de 1807 sólo hay seis cuarteles donde residen criollos mientras que en el padrón de agosto de 1810 figuran en la totalidad de los cuarteles, es decir catorce. También hay que considerar que el número total de españoles descendió 6.485 personas en 1810.

El descenso demográfico español no parece que tenga su causa en la emigración o muerte biológica, sino en otros motivos. Esto nos lleva a proponer aceptando las observaciones en documentos británicos- la hipótesis de "muerte ciudadana”. Luego del avance francés en España, la disolución de la Junta de Sevilla y la triunfante revolución de Mayo de 1810, muchos españoles porteños simpatizaron con 
la causa americana. Con el nuevo gobierno de la Primera J unta estuvieron dispuestos a formar parte de las filas patriotas, adoptando la ciudadanía criolla. ${ }^{4}$

\title{
El Comercio y Otras Profesiones
}

En la ciudad de Buenos Aires las profesiones eran ejercidas por individuos con una determinada ubicación en la escala social. Incluso de acuerdo a una división de carácter racial. Los oficios manuales eran realizados por personas de piel más oscura, según el grado de mestizaje:

\begin{abstract}
los españoles y los criollos blanqueados dejan tales ocupaciones para sus paisanos más oscuros, que son industriosos en sus respectivos oficios como zapateros, sastres, barberos, changadores, pulperos, carpinteros y pequeños comerciantes al menudeo. (GILLESPIE, 1994, p. 53).
\end{abstract}

Sin embargo, también los británicos ejercieron algunos de estos oficios despreciados por la gente blanca española o americana. En 1804 había un carpintero, dos sastres, dos zapateros y un herrero de origen británico, siendo estas actividades consideradas, por la sociedad porteña, para gente de piel oscura. Evidentemente estos oficios eran muy demandados y -en algunos casos- bien remunerados. "Los oficios de zapateros y sastres, los últimos principalmente mujeres, son los más numerosos y ocupados." (GILLESPIE, 1994, p. 85). Por esto mismo se entiende que algunos británicos no tuviesen inconvenientes en ejercerlos ya que eran competentes en estas profesiones. En particular el oficio de herrero tenía especial importancia. "Había solamente dos herreros en la ciudad, siempre muy morosos en sus obras, pero sólidas en su conclusión." (GILLESPIE, 1994, p. 54).

En 1804 estaba registrado un maestro herrero de origen irlandés, Alexandro Guillermo. Los datos indican que era casado con "una inglesa" y poseía dos esclavos, oficiales herreros. En 1809 Guillermo figura empadronado en el Cuartel número 5, por lo tanto podemos inferir que éste sería uno de los dos herreros que Gillespie dice haber visto en 1806. Curiosamente Alexandro Guillermo no figura en el padrón de 1807. En el padrón de 1809 reaparece como inglés y casado con Isabel Gel "de la propia nación". Ante la contradictoria nacionalidad surgida de los datos de dos padrones (1804-09) la duda que surge es: ¿Era irlandés o inglés? Afortunadamente el empadronador de 1809 abundó en algunos datos sobre A. Guillermo. Llegó a Buenos

\footnotetext{
4 The people here are in effect unison with their lawful Sovereing Ferdinand the VII; but should the Usurper prove successful in conquering the Peninsula, [...] these inhabitants and the Government too, will consider their connection with the mother country as totally dissolved. Cfr. Carta a George Canning, 10/9/1809. Ravignani (1964, p. 187).
} 
Aires prisionero, al igual que su esposa, en la Fragata Ladisor, sin precisar el año ni la causa de su prisión, ni la de su esposa. Pero sí sabemos que "se reconciliaron con la Iglesia en esta Ciudad", por lo tanto consideramos que eran ingleses y que el censista de 1804 cometió un error al calificarlo de "irlandés", ya que todos los irlandeses registrados en estos censos eran católicos.

De los datos censados en 1804 surge que entre los británicos casados no hay ningún comerciante. Todos ejercen oficios: zapatero, sastre, medico, herrero, piloto de altura, carpintero, botero. Los comerciantes son solteros (exceptuando a un viudo, irlandés y negrero, Felipe Reilly). Podríamos arriesgar con fundamento que esa actividad se asemejaba mucho a la de los aventureros y marinos ya que eran viajeros, con largas travesías por los mares del mundo. Los desembarcos daban relativa estabilidad de residencia, pero estaban sujetos a los permisos transitorios otorgados por las autoridades españolas.

Cuadro 2- Empadronamiento Año 1804

\begin{tabular}{|l|c|c|c|c|c|}
\hline CUADRO 2 & \multicolumn{5}{|c|}{ EMPADRONAMIENTO ANO 1804 } \\
\hline & tot. extranjeros & británi & \% & com. Brit. & \% \\
\hline cuartel 2 & 17 & & & & \\
\hline cuartel 3 & 39 & & & & \\
\hline cuartel 4 & 50 & 2 & & & \\
\hline cuartel 5 & 59 & 5 & & 3 & \\
\hline cuartel 6 & & 1 & & & \\
\hline cuartel 7 & 62 & 7 & & & \\
\hline cuartel 8 & 24 & 3 & & 2 & \\
\hline cuartel 9 & 19 & 4 & & 1 & \\
\hline cuartel 12 & 14 & & & & \\
\hline cuartel 13 & 5 & 3 & & & \\
\hline cuartel 14 & 20 & 3 & & & \\
\hline cuartel15y18 & 13 & & & & \\
\hline cuartel 16 & 17 & & & & \\
\hline cuartel 19 & 14 & & & & \\
\hline cuartel 20 & 11 & & & & \\
\hline total & $\mathbf{3 6 4}$ & $\mathbf{2 8}$ & $\mathbf{7 , 6 9}$ & $\mathbf{6}$ & $\mathbf{2 1 , 4 3}$ \\
\hline
\end{tabular}

Con la ocupación comerciante figuran en el censo de 1804 seis británicos. Los comerciantes (21,43\% del total) constituían la principal actividad entre los británicos residentes en Buenos Aires ese año. Los médicos -tres en total- le seguían entre las ocupaciones más numerosas. Luego los pilotos, boteros, zapateros y sastres, con dos trabajadores por cada una. Terminaba la lista el único maestro herrero británico, el mencionado Alexandro Guillermo, quien con esfuerzo había alcanzado el 
respeto de la sociedad porteña. Llegado como prisionero en la Fragata Ladisor se reconcilió con la iglesia católica -junto a su esposa- y vivió "en buena armonía sin dar motivos al vecindario y es uno de los mejores y equitativos herreros". Sin lugar a dudas que le iba bien en su negocio ya que tenía "esclavos, taller público y casa de su propiedad", en Alto de San Pedro.

En este censo también hay un británico de apellido Gorman, de profesión "protomédico", quien "vive con dos inglesas". En el censo de 1809 el empadronador registra su nombre completo: Miguel Gorman," irlandés, protomédico de 71 años". De acuerdo a otras lecturas sabemos que este médico arribó a Buenos Aires en 1777, como cirujano de la real armada, en la expedición de don Pedro de Ceballos, primer virrey del Virreinato del Río de la Plata. En 1779, por Real Orden, lo designan para reorganizar el hospital y se radica definitivamente en Buenos Aires. Fue el primer médico y fundador de la Escuela de Medicina. (BATTOLLA, 1928, p. 71).

Sobre el número elevado de británicos ocupados en el comercio conviene aclarar que "Aquí la honorable denominación de comerciante está tristemente pervertida, como que implica, sin distinción, el primer negociante y el ínfimo revendedor". (GILLESPIE, 1994, p 85.). En 1804 encontramos a tres comerciantes que se dedicaban al "cargamento de negros": J osé Maló (inglés), Felipe Reilly y Thomas O Reilly (ambos irlandeses) También había un irlandés comerciante de pieles, Daniel Donnoghue, en cuya casa vivían varios británicos (J uan de la Puente y esposa, Juan Marcos y Guillermo Jones). De todos modos consideramos que el número de británicos, y entre éstos los comerciantes, era mayor al registrado en los censos. A modo de ejemplo mencionamos a Diego Williamns quien es citado en el padrón de 1804, pero no figura empadronado (por lo tanto no lo contamos). Sólo podemos inferir su nacionalidad británica y ocupación comerciante porque el censo citado sí registra a Guillermo Jones, "soltero, inglés de religión anglicana", donde señala que era "dependiente" de Williams. ${ }^{5}$ Además Gillespie (1994, p. 40) menciona que hubo "muchos prisioneros comerciantes caídos en manos del enemigo después de la reconquista, que fueron abandonados y olvidados". Esta afirmación sirve para explicar porqué en el padrón de febrero de 1807 disminuyó más del $50 \%$ el número de británicos y porqué no se registró ningún comerciante de esa nacionalidad (Ver Cuadro 3). El éxodo de los comerciantes fue extraordinariamente grande porque durante la invasión de 1806

${ }^{5}$ Padrón 1804 en Documentos para la Historia Argentina (RAVIGNANI, 1964, p. 152, t. 12). 
Había más de seiscientos tenderos en Buenos Aires, que vendían todos los artículos como nuestros. Sus mercaderías eran generalmente lo más ordinarias, habiéndose suspendido hacía mucho tiempo el tráfico con Europa en cualquier escala, y sus auxilios fabriles eran suministrados por las toscas manufacturas del Perú o los algodones de los Brasiles. (GILLESPIE, 1994, p 85.).

No podemos confirmar, según los datos proporcionados por el censo de 1807, el número de profesiones existentes en la ciudad. Pero sí es nuestro objeto analizar las variaciones del número de comerciantes británicos entre los censos de 1804, 1807 y 1810. Es importante saber que la profesión "comerciante" abarcaba un amplio espectro de actividades."Los principales comerciantes tienen comisionistas viajeros, como los nuestros, por todo el país." (GILLESPIE, 1994, p 85.). Conviene señalar que luego de la reconquista los comerciantes británicos transeúntes huyeron. Además muchos soldados del Reino Unido desertaron y se encontraban alojados como peones en quintas de los alrededores de la ciudad de Buenos Aires. Así lo manifiesta el alcalde del cuartel número10.

Regente y oidores dela R. Audiencia Governadora delas Provincias del Rio dela Plata

En obedecimiento del vando q. se publicó ayer 4 del corr. En esta ciudad, sobre q. Los vecinos de ella dén parte alos respectivos Alcaldes de Barrio delos ingleses y demas extranjeros q tengan en sus Casas, debo decir a Vm- que en mi chacra ó quinta, distante de esta Ciudad como dos leguas, tengo un muchacho irlandes o ingles de los que se desertaron en los próximos dias dela reconquista trabajando en calidad de peon asalareado: lo que pongo en noticia de $\mathrm{Vm}$ para q inmediatamente dé parte a donde corresponde, afin de q en ningun tiempo me prepare perjuicio.

Dios guarde a Vm, m a. Buenos Ayres 5 de abril de 1807

Diego J ose de Sosa. (RAVIGNANI (1919, p. 260).

El temor a una nueva e inminente invasión (1807) hizo tomar medidas de seguridad, expulsando potenciales espías einformadores británicos.

\section{Empadronamiento de 1807}

La realización del empadronamiento de los extranjeros residentes en la ciudad de Buenos Aires, en el mes de febrero del año 1807, tuvo estrecho vínculo con el pedido de rendición al gobierno capitalino, realizada por los ingleses el 26 de febrero de 1807. La sospecha de que podía haber conspiradores o espías entre los extranjeros alojados en las familias porteñas puede desprenderse de la siguiente afirmación de Gillespie (1994, p. 82). 


\begin{abstract}
Después que estábamos prisioneros muchas de las familias de la ciudad mostraron un deseo especial de tener soldados ingleses como domésticos, mucho más por el deseo liberal de aliviar su cautividad, que para beneficiarse con su servicio. En estos empleos nuestros subordinados también participaban de sus bondades, y existía evidente preferencia por parte de las mujeres hacia los oficiales ingleses, que la demostrada a esa clase de sus amigos.
\end{abstract}

Las buenas relaciones con algunas familias iba más allá de los aspectos sociales, con implicancias políticas y comerciales. También hay que tener en cuenta que en 1806 prácticamente no había ejército español para la defensa.

Todos los establecimientos militares de España en aquellas regiones remotas habían mermado hasta convertirse en gentuza, debido a la falta de refuerzos de la península, y las únicas tropas que vi dignas de tal nombre no excedían de ciento treinta hombres, a quienes se les aplicaba el título de veteranos. (GILLESPIE, 1994, p 87.)

Además, Gillespie refuerza nuestra argumentación considerando que el alistamiento del ciudadano común - para lo cual era importante el empadronamientoanticipó la formación del ejército patriota.

como cada soldado en pie, para el propósito de la defensa común, era nativo del suelo, debe haber sido evidente que esos defensores fueran los primeros en alistarse bajo banderas. Esa es la explicación radical de aquellas facilidades con que Buenos Aires se ha convertido en estado independiente. (GILLESPIE, 1994, p 87.).

Matheo Correa figura como "islandés artillero de W." Posiblemente se trata de un error y correspondería leerse "irlandés", similar al caso encontrado en el censo de 1804. Sobre la ocupación "artillero de W." podría significar "artillero de Werresford", deformación del apellido Beresford, realizada por el censista, que también observamos en el censo de 1809, cuartel número10. En el censo de 1807 se registran cuatro británicos quienes podrían ser solamente dos. Norberto de Brorrié y Roberto Brorie talvez fueron la misma persona ya que poseían la misma edad y uno estaba en poder de Alvarez y otro de Abarres (deformación de apellido que ya observamos en otro lugar). Además, Jaime Egans podría ser J aime Erzano, ambos irlandeses, herreros, solteros y censados en el mismo cuartel. De esta manera también coincidiría el relato de Gillespie: durante la conquista sólo había dos herreros en la ciudad. Ellos, según los censos, serían Alexandro Guillermo y Jaime Egans, aunque curiosamente el primero no aparece en 1807. De acuerdo a esta reducción, de cuatro personas en dos, tendríamos un total de diez británicos en Buenos Aires (1807) Los porcentuales según su estado civil serían: 


\section{Solteros 5 (50 \%) \\ Casados 4 (40\%) \\ Sin datos $1(10 \%)$}

Estas cifras indican casi la misma proporción entre casados y solteros que en el censo de 1804, excepto por una ligera mayoría de casados y una notable disminución del $30 \%$ de los Sin datos (aquellos que no tienen registrada su situación civil) También llama nuestra atención el censo de un extranjero de nombre Guillermo Russel ¿Será el mismo Mr. Russel, escocés, señalado por Gillespie (1994, p. 31), "súbdito naturalizado de Buenos Aires, después de una residencia de años"? Lamentablemente no hay más datos en el censo. Tampoco Gillespie menciona su nombre completo, sólo que fue de extraordinaria importancia en la conquista de Buenos Aires. Primeramente porque Russel habría dado a los ingleses motivación para obtener riqueza segura y con poco riesgo, debido a su informe de la débil defensa de la ciudad:

La noticia dada por Mr. Russel, fue que una gran suma de dinero había llegado a Buenos Aires desde el interior para ser embarcada rumbo a España en la primera oportunidad, que la ciudad estaba protegida solamente por una poca tropa de línea, cinco compañías de indisciplinados blandengues, canalla popular, y que la festividad de Corpus Christi, que se aproximaba y atraía la atención de todos, terminando en una escena de borrachera general y tumulto, sería la crisis más favorable para un ataque contra la ciudad. (GILLESPIE, 1994, p.31-32).

Finalmente, este británico gozaba ampliamente de la confianza española porque "desempeñaba el puesto de práctico real en el Plata". Y supo guiar a los ingleses por el río hasta llegar "frente a Quilmes". ${ }^{6}$

Adjuntamos el siguiente cuadro a los efectos de poder comparar la situación de los comerciantes británicos con sus pares registrados en otros padrones. Es significativa la ausencia de este grupo profesional - ese año- y el escaso número porcentual de británicos en relación al total de extranjeros.

\footnotetext{
${ }^{6}$ Los servicios de Russel "fueron pobremente recompensados" por los ingleses y al regresar a Buenos Aires "fue encarcelado por los españoles cuando la reconquista de la ciudad". Ver Gillespie, (1994, p 31-33).
} 
Cuadro 3- Empadronamiento año 1807

\section{EMPADRONAMIENTO AÑO 1807}

\begin{tabular}{|l|c|c|c|c|}
\cline { 2 - 5 } \multicolumn{1}{c|}{} & tot extranjeros & británic & \% & co. Brit. \\
\hline cuartel 1 & 4 & 0 & & 0 \\
\hline cuartel 2 & 18 & 0 & & 0 \\
\hline cuartel 3 & 30 & 0 & & 0 \\
\hline cuartel 4 & 25 & 1 & & 0 \\
\hline cuartel 5 & 49 & 2 & & s/d \\
\hline cuartel 6 & 37 & 1 & & 0 \\
\hline cuartel 7 & 57 & 0 & & 0 \\
\hline cuartel 8 & 34 & 1 & & s/d \\
\hline cuartel 9 & 23 & 1 & & 0 \\
\hline cuartel 10 & 3 & 1 & & 0 \\
\hline cuartel 12 & 6 & 0 & & 0 \\
\hline cuartel 13 & 35 & 0 & & 0 \\
\hline cuartel 14 & 23 & 1 & & 0 \\
\hline cuartel 15 & 15 & 0 & & 0 \\
\hline cuartel 16 & 9 & 0 & & 0 \\
\hline cuartel 17 & 11 & 0 & & 0 \\
\hline cuartel 18 & 10 & 1 & & 0 \\
\hline cuartel 19 & 6 & 0 & & 0 \\
\hline cuartel 20 & 2 & 1 & & 0 \\
\hline total & $\mathbf{3 9 7}$ & $\mathbf{1 0}$ & $\mathbf{2 , 5 2}$ & $\mathbf{0}$ \\
\hline
\end{tabular}

\section{Empadronamientos de 1809 y 1810}

Luego de la segunda invasión británica al Río de la Plata (1807) y de la nueva reconquista de Buenos Aires por parte de su población, vino otra orden de empadronamiento de residentes extranjeros, en Agosto de 1809. El número de británicos había ascendido hasta 70 individuos como mínimo (Ver Cuadro 5). La cifra real es difícil precisar porque el censo del cuartel número 8 (a cargo del alcalde Manuel Ventura de Haedo) señala que en la Calle de Unquera hay una casa "en que habitan varios negociantes ingleses con almazen de efecto de su nación". (RAVIGNANI, 1919, p. 289) Como esta información no da el número exacto de ingleses hemos resuelto colocar el número mínimo de 3. J ustificamos la colocación de esta cantidad porque suponemos que si hubieran sido dos los individuos el censista hubiera escrito "dos" y no "varios", dando a entender que se trataba de una cantidad relativamente superior a dos. Por este motivo colocamos un número aproximado de 4 ingleses en dicho cuartel (un talabartero y tres comerciantes, como mínimo).

Por otra parte, muchos ingleses o extranjeros no eran censados, así lo comprobamos cuando confrontamos la carta firmada el 20 de Diciembre de 1809 por 
varios comerciantes británicos residentes en Buenos Aires. Cinco de ellos no aparecen en el censo del mismo año. Además, si tenemos en cuenta los informes del Southern Star el número total de británicos talvez fuera superior a 70. Al menos considerando los comerciantes en tránsito. Para reafirmar la existencia de residentes británicos sin registrar transcribimos un informe del cuartel número 9, a cargo del alcalde Rafael Blanco:

Acompaño a VE la razon de los extranjeros que habitan en el cuartel $\mathrm{n}^{\circ} 9$ de mi cargo en cumplimiento de la onden que VE se sirvió comunicar el 3 del corriente a los Alcaldes de barrio

Dios nro. Sor.gue a VE m.s a.s

Rafael Blanco

Buenos Ayres Agosto 8 de 1809

Luego de señalar el nombre de los extranjeros resguarda la identidad de los británicos.

Tres sujetos en casa de D J ose Leon Viruti Manzana 81 reputados por ingleses cuyos nombres y exercicio no se ha podido averiguar con reserva

(y al final del listado) No se ha podido tomar razon puntual de los nombres y apellidos de varios sujetos de esta lista por guardar la reserva que se ha encargado

Rafael Blanco ${ }^{7}$

La reserva puede deberse a un pedido de la autoridad superior. Esto, como veremos más adelante, quizá guarda relación con gestiones llevadas ante el mismo virrey por el jefe de la escuadra naval británica. Podría ser una práctica relativamente habitual que se pidiera la reserva de los nombres de aquellos británicos con vencimiento de su residencia, pero que estaban gestionando una prorroga. En definitiva, de un total de 70 británicos en Buenos Aires (1809) las variaciones porcentuales según su estado civil son:

\author{
Solteros $25(35,7 \%)$ \\ Casados $32(45,7 \%)$ \\ Sin datos $13(18,6 \%)$
}

Estas cifras muestran una clara tendencia a la búsqueda de constituir familia en Buenos Aires, por parte de los británicos transeúntes. Por primera vez, desde 1804, los casados superan en porcentaje a los solteros británicos residentes en Buenos Aires. Además, considerando únicamente los comerciantes (18) que figuran en el censo de

${ }^{7}$ Padrón de 1809. 
1809, éstos representan el 25,71 \% del total de los británicos (70). El porcentaje es superior a la proporción de comerciantes existentes en 1804. Si analizamos el número de comerciantes de 1809 en función de la mercadería ingresada durante la primera invasión vemos que:

1) Junto a la invasión militar se dio otra invasión comercial inglesa con productos de ese origen, pero de mala calidad, lo cual influyó negativamente en la inserción de comerciantes y en la venta de sus productos debido a la desconfianza.

Una especulación de relojes de bolsillo, si son con tapa y adornados con piedras preciosas, y llevan la marca de un fabricante inglés, raramente fallará en una escala limitada, pero deben ser de bondad garantida. Debido al rechazo de estas, como de otras mercaderías que habían sido derramadas sobre Buenos Aires algún tiempo después de su conquista por emigrantes necesitados de Inglaterra, poco cuidadosos de la reputación nacional, una prevención considerable existió contra todo artículo con marcas de su manufactura por largo tiempo subsiguiente a la reconquista del lugar. (GILLESPIE, 1994, p 54).

2) Aumentó el factor de incertidumbre entre los mercaderes británicos residentes. Esto a pesar de la alianza británica con España (1808) y del Acta del 6 de noviembre de 1809 sobre libre comercio, firmada por el virrey Baltasar Hidalgo de Cisneros. ${ }^{8}$

En la mencionada Acta se concedía a los comerciantes un derecho de residencia, pero cuya duración estaba limitada en el tiempo. Por lo tanto, la situación de la residencia de los mercaderes ingleses era precaria y pendía sobre ellos el vencimiento de los permisos. Al finalizar tenían que ser renovados o retirarse del país. Fue entonces cuando los comerciantes británicos, dirigidos por Alexander Mackinnon, formaron un comité. El motivo era realizar una solicitud escrita a Sir Bentick C. Doyle, comandante de la flota británica al mando del H. M. S. Lightning. El pedido consistía en que

\footnotetext{
${ }^{8}$ El comerciante Alexander Mackinnon escribía a George Canning, cónsul británico en Brasil: "Buenos Aires, 10-12-1809. Tengo el agrado de transmitir a V:E: una copia y traducción del manifiesto del Virrey del 2 de noviembre y del decreto de la J unta fechado el 6 , promulgando a raíz del primero, estableciendo las directivas, reglamentaciones e impuestos que deben ser observados en el intercambio comercial autorizado mediante él por este gobierno con Gran Bretaña [...]. El manifiesto del virrey pinta en colores más subidos de lo que me aventuraría a escribir, el estado de este país, y las urgencias que forzaron al gobierno a buscar una renta mediante el comercio directo (not a free trade as it is very improperly colled) con Gran Bretaña. [...] se me ha asegurado que ninguna persona de buen carácter que tenga verdaderos negocios que realizar o una profesión legítima que ejercer y que se conduzca pacífica e inofensivamente, será molestada. Estas son sólo palabras, lo otro es una ley escrita, y la situación de los súbditos británicos, aun de aquellos de conducta más correcta, es muy precaria hasta que algún acuerdo mutuo entro los dos gobiernos haga nuestro intercambio más seguro, Ravignani (1962, p. 184)
} 
mediara ante el Virrey Cisneros, para que no se efectivizara la orden de expulsión notificada a algunos de ellos.

Este es el contenido de la carta presentada el 20 de diciembre de1809: ${ }^{9}$

Varios de nosotros, los abajo firmantes, comerciantes británicos que residen en Buenos Aires en la actualidad, hemos recibido inesperadamente notificaciones del gobierno para que efectuemos nuestra partida en el breve período de ocho días, y nos permitimos dirigirnos a V.E. sobre un asunto en el que tenemos tanto interés, y solicitar de V. E. intervenga por nosotros . Es bien sabido que hace algún tiempo Su Excelencia el Virrey concedió permiso a los navíos británicos para introducir cargamentos de mercadería en este río contra el pago de ciertos derechos, bajo la confianza de este permiso muchos de nosotros hemos descargado y desembarcado mercaderías haciéndolas pasar en forma reglamentaria por la Aduana, en tanto otros confiados en que continuará en vigor el permiso otorgado, han encargado otras mercaderías cuya llegada puede producirse de un momento a otro. En otros casos tenemos sumas adeudadas a nosotros, cuyo cobro requerirá algún tiempo; por todas estas circunstancias, no escapará en consecuencia a la atención de V. E. el inconveniente de estar obligados a abandonar un lugar donde tenemos tantos intereses comprometidos, confiando naturalmente en que tras una adecuada presentación de los hechos a Su Excelencia el Virrey, él tendrá a bien permitir nuestra residencia en esta ciudad por el tiempo que sea necesario para nuestros negocios. Nosotros declinamos toda interferencia en política o en asuntos públicos, y por ello aquí declaramos que nuestra intención de residir en esta ciudad solamente obedece al manejo de nuestros intereses privados.

Rogamos a Ud. nos haga el favor de llevar esta circunstancia a conocimiento de su Excelencia el Virrey, y obtener de él una respuesta tan pronto como sea conveniente,

Tenemos el honor de ser, Señor, sus más obedientes servidores.

Alexander Mackinnon, Thomas Crokett, Edward Hill, James Ritchie, J ames Barton, J onas T. Smedly, William Dunn, George Dyson, Frederick Dowling, J. Rattray

Finalmente, en la búsqueda del crecimiento de la población de Buenos Aires y del número de comerciantes ingleses confeccionamos el Cuadro 10. Este se realizó de acuerdo a los datos que figuran en los "resúmenes de los padrones generales que manifiestan en número de habitantes existentes", en los cuarteles del padrón de agosto de 1810. Pero nos encontramos con la sorpresa que allí no registran el número de británicos. Por lo tanto, en primer lugar realizamos el análisis comparativo de la población, sin considerar a los británicos. En 1810, con sólo 14 cuarteles censados, Buenos Aires alcanzaba 31.977 habitantes. En 1807, con 10 cuarteles completos, totalizaba 21.313 habitantes (Cuadro 9) Pero, si sumamos sólo aquellos cuarteles completos y los comparamos, año por año, vemos que (Cuadro 4) la población se había mantenido casi estable.

\footnotetext{
${ }_{9}^{9}$ Copia del oficio que contiene la petición de los comerciantes británicos al Virrey en Mayo Documental. (RAVIGNANI, 1964, p. 225).
} 
Cuadro 4- Población de B. Aires

\begin{tabular}{|l|c|c|}
\hline \multicolumn{3}{|c|}{ Población de B. Aires } \\
\hline Año & $\mathbf{1 8 0 7}$ & $\mathbf{1 8 1 0}$ \\
\hline Cuartel 2 & 2153 & 2280 \\
\hline Cuartel 5 & 4450 & 4237 \\
\hline Cuartel 6 & 2878 & 2519 \\
\hline Cuartel 11 & 1046 & 1313 \\
\hline Cuartel 13 & 2838 & 2738 \\
\hline Cuartel 17 & 1874 & 2064 \\
\hline Total & $\mathbf{1 5 2 3 9}$ & $\mathbf{1 5 1 5 1}$ \\
\hline
\end{tabular}

Los extranjeros incrementaron su número en 75, respecto a 1807, pero el porcentaje sobre el total de la población disminuyó ligeramente, 0,41\% (Comparar Cuadros 9 y 10). En segundo lugar, debido a la ausencia de datos sobre británicos, en 1810, para hacer un análisis comparativo nos vimos obligados a considerar las cifras obtenidas en 1809. ${ }^{10}$ Allí los comerciantes británicos representaron el 17\% del total de residentes de ese origen. Este porcentaje muestra un importante ascenso, luego de la caída de 1807. Pero no alcanza el nivel de 21,43 \%, logrado en 1804 (Cuadros 5, 3 y 2).

Cuadro 5- Empadronamiento año 1809

\begin{tabular}{|c|c|c|c|c|c|}
\hline \multirow[t]{2}{*}{ Cuartel } & \multicolumn{5}{|c|}{ EMPADRONAMIENTO AÑO 1809} \\
\hline & \begin{tabular}{|c|} 
Total \\
extranjero \\
$\mathbf{S}$
\end{tabular} & británico & $\%$ & $\begin{array}{l}\text { com. } \\
\text { Brit. }\end{array}$ & $\%$ \\
\hline cuartel 2 & 8 & 0 & & 0 & \\
\hline cuartel 3 & 45 & 17 & & 0 & \\
\hline cuartel 4 & 33 & 3 & & 0 & \\
\hline cuartel 5 & 73 & 9 & & 0 & \\
\hline cuartel 6 & 24 & 4 & & 2 & \\
\hline cuartel 7 & 48 & 20 & & 7 & \\
\hline cuartel 8 & 37 & 4 & & 3 & \\
\hline cuartel 9 & 21 & 3 & & 0 & \\
\hline cuartel 10 & 5 & 0 & & 0 & \\
\hline cuartel 12 & 8 & 2 & & 0 & \\
\hline cuartel 13 & 46 & 6 & & 0 & \\
\hline cuartel 14 & 21 & 1 & & 0 & \\
\hline cuartel 15 & 12 & 0 & & 0 & \\
\hline cuartel 16 & 9 & 0 & & 0 & \\
\hline cuartel 18 y19 & 11 & 0 & & 0 & \\
\hline cuartel 20 & 7 & 1 & & 0 & \\
\hline total & 408 & 70 & 17,15 & 12 & 17,1 \\
\hline
\end{tabular}

${ }^{10}$ Las variaciones en el número total de extranjeros son: 364 (1804), 397 (1807) , 408 (1809) y 472 (1810).

Las variaciones en el número total de comerciantes británicos: 6 (1804), 0 (1807) y 12 (1809). 


\section{Conclusiones}

Después de la investigación realizada estamos en condiciones de responder algunos de los interrogantes que nos habíamos formulado, y otros que surgieron en el transcurso del trabajo.

¿Con qué fin vinieron los primeros británicos a Buenos Aires? Utilizando las herramientas documentales disponibles podemos sugerir que hubo dos grandes grupos:

a) Comerciantes y aventureros

b) Perseguidos y redimidos

Entonces, la pregunta sobre los fines para los que vinieron los británicos a Buenos Aires se responde por las actividades que realizaron. Cuantitativamente se puede afirmar que, en 1804, entre los que constituyeron el primer grupo la mitad eran negociantes de esclavos y el resto comerciantes transeúntes de manufacturas. La actividad del comercio de esclavos era importante no sólo entre los británicos, también los portugueses la realizaban en nuestra ciudad. Evidentemente quienes ejercían esa actividad sólo tenían residencia temporal.

El segundo grupo lo constituyeron británicos con problemas legales que estuvieron dispuestos a redimirse (ingresar a la Iglesia católica) o bien eran católicos irlandeses. Obtenida la residencia, desempeñaron actividades donde no competían con los españoles, y a su vez eran relativamente bien remuneradas, para aquellos que sabían realizarlas (zapateros, herreros, sastre). El mejor ejemplo lo obtenemos de los únicos dos herreros de la ciudad, quienes eran británicos. En el padrón de 1807 observamos una disminución del número de británicos en más del 50\% y la ausencia de sus comerciantes. Esto se explica porque - luego de la reconquista de Buenos Airesmuchos de ellos fueron hechos prisioneros, según afirma Gillespie, y otros -para no correr la misma suerte- habrán huido.

¿Cuáles fueron sus vínculos con la sociedad porteña? Las mayores dificultades que tenían los ingleses para integrarse socialmente provenían de dos factores que los diferenciaban: el idioma y la religión. Además, a excepción del momento de la invasión inglesa de 1806, había una mayoría de hombres españoles en Buenos Aires. Por ese motivo los matrimonios se realizaban entre miembros de la misma comunidad de origen. En 1804, de 6 casados 4 constituían matrimonios intercomunitarios. Si incorporamos a ese grupo a los 2 viudos, el 75\% \% de los casamientos eran entre británicos. Pero en 1809 la situación estaba cambiando hacia la formación de matrimonios mixtos. Ese año, de 32 ingleses casados únicamente 10 lo hicieron 
comunitariamente. $\mathrm{Si}$ sumamos dos más -porque "viven juntos"- tenemos que los matrimonios intracomunitarios constituían sólo el 37,5 \%. Los 45 días de dominación inglesa en 1806 influyeron positivamente en la tendencia a la formación de matrimonios mixtos. El relato de Gillespie es muy claro: muchos jóvenes británicos fueron prisioneros (o desertores) trabajando en familias patricias, y las jóvenes criollas tenían preferencia por esos oficiales. Evidentemente que fue mas fácil la integración con los irlandeses, por ser católicos. Los censos posteriores a 1804 no ofrecen datos sobre la religión. Pero el empadronamiento de 1809 indica que al menos dos casamientos entre ingleses ocurrieron con el ingreso a la Iglesia católica, lo cual es señal de integración de los anglicanos a la sociedad porteña. Estos constituían el grupo de "perseguidos y redimidos", quienes ejercieron sus oficios (zapateros, herreros, curtidores) y consiguieron residencia como respetables vecinos.

El grupo de los "comerciantes y aventureros" no consiguieron residencia fácilmente, pero -en esa búsqueda- tuvieron mayor vinculación con la clase dirigente del virreinato. Esto se dio principalmente luego de las frustradas invasiones inglesas a Buenos Aires. En 1808, por las cambiantes situaciones de la política internacional, el Reino Unido viró de enemigo a aliado de España. A partir de ese momento los comerciantes recurrieron a sus vínculos políticos para conseguir una residencia prolongada en Buenos Aires. En este punto surge un nuevo interrogante:

¿Influyeron los comerciantes británicos en el levantamiento patriota de Mayo de $1810 ?$

La documentación británica citada indica que la medida tomada por Cisneros el 2 de noviembre de 1809, sobre comercio (no libre, sino directo) con Gran Bretaña, tenía objetivos fiscales. Sólo buscaba aumentar los ingresos aduaneros proveniente de las mercaderías británicas. Por lo tanto, las autoridades españolas no iban a cambiar la vieja prohibición de residencia que regía sobre los extranjeros en el Virreinato. Esta situación era contradictoria para los comerciantes británicos. Por una parte querían establecerse en Buenos Aires para hacer sus negocios, pero Cisneros continuaba siendo el principal obstáculo para una residencia prolongada. El último plazo de permanencia vencía el 18 de Mayo, luego debían retirarse. Por este motivo, y por la apertura plena del comercio, habrían apoyado al movimiento revolucionario que derrocó al virrey y permitió su anhelada residencia. La presión política ejercida por los comerciantes es manifiesta en la carta dirigida al Comandante de la flota británica. Allí se constituyó el primer comité y se realizó la primera operación política en defensa de los "intereses privados" británicos en Buenos Aires. Indicios documentales señalan que no fue la 
última, antes y después de la Revolución de Mayo. ${ }^{11}$

Finalmente podemos afirmar que, de acuerdo a la hipótesis planteada, las invasiones inglesas favorecieron el interés de los británicos por Buenos Aires. Esto generó un aumento del $150 \%$ en el número de sus residentes (1804-1809). Los comerciantes, continuaron siendo la principal actividad, 17\%. Aunque el número de ingleses censados en 1809 sin más datos, por "reserva" oficial, sugiere un número mayor de comerciantes cuya residencia estaba vencida.

${ }^{11}$ No adjuntamos esa documentación porque excedería el espacio del presente trabajo. Nos referimos a cartas británicas y resoluciones de la Primera J unta de Gobierno patrio. 
Cuadro 6- Empadronamiento nov-dic 1804

\begin{tabular}{|c|c|c|c|c|c|}
\hline \multirow{3}{*}{\begin{tabular}{|l} 
Cuartel \\
cuartel 4
\end{tabular}} & \multirow{3}{*}{\begin{tabular}{|l|} 
nombre \\
M de los Angeles \\
Garate de Elezmar \\
\end{tabular}} & \multicolumn{3}{|c|}{ EMPADRONAMIENTO NOV-DIC 1804} & \multirow[b]{2}{*}{ ocupación } \\
\hline & & \multicolumn{2}{|c|}{ nacionalidad religión } & \multirow{2}{*}{\begin{tabular}{|l|} 
est civil \\
casada
\end{tabular}} & \\
\hline & & inglesa & $s / d$ & & $\mathrm{~s} / \mathrm{d}$ \\
\hline & Roberto Calaau & irlandés & católico & viudo & zapatero \\
\hline \multirow[t]{5}{*}{ cuartel 5} & Diego Maló & ingles & católico & soltero & negrero \\
\hline & David Reid & escocés & católico & cas c/ esp & médico \\
\hline & Alexandro Guillermo & irlandés & católico & c c/ inglesa & herrero \\
\hline & Thomas O Reilly & irlandés & católico & $\mathrm{s} / \mathrm{d}$ & negrero \\
\hline & Felipe Reilly & irlandés & católico & viudo & negrero \\
\hline cuartel 6 & J orge Aliburcon & escocés & católico & c c/ inglesa & piloto de altura \\
\hline \multirow[t]{7}{*}{ cuartel 7} & Luisa Ricardo & ingles & $s / d$ & $\mathrm{~s} / \mathrm{d}$ & $\mathrm{s} / \mathrm{d}$ \\
\hline & J osefa Clara & ingles & $\mathrm{s} / \mathrm{d}$ & $\mathrm{s} / \mathrm{d}$ & $\mathrm{s} / \mathrm{d}$ \\
\hline & Juana Grin & ingles & $\mathrm{s} / \mathrm{d}$ & $\mathrm{s} / \mathrm{d}$ & $\mathrm{s} / \mathrm{d}$ \\
\hline & J aime Vidal & islandés & católico & c c/ inglesa & carpintero \\
\hline & Gorman & irlandés & $\mathrm{s} / \mathrm{d}$ & $\mathrm{s} / \mathrm{d}$ & protomédico \\
\hline & Dos inglesas viven & inglesa & $\mathrm{s} / \mathrm{d}$ & $\mathrm{s} / \mathrm{d}$ & $\mathrm{s} / \mathrm{d}$ \\
\hline & c/ Gorman & inglesa & & & \\
\hline \multirow[t]{3}{*}{ cuartel 8} & Diego J acson & ingles & católico & soltero & comerciante \\
\hline & Juan Bene & ingles & protestante & soltero & sastre \\
\hline & Florencio Maccarth & irlandés & católico & soltero & comerciante \\
\hline \multirow[t]{4}{*}{ cuartel 9} & Guilllermo J ones & ingles & anglicano & $\mathrm{s} / \mathrm{d}$ & dependiente \\
\hline & Juan de la Puente & $\mathrm{s} / \mathrm{d}$ & católico bautizado & c c/inglesa & botero \\
\hline & Daniel Donnoghue & irlandés & católico & soltero & comerc pieles \\
\hline & Juan Marcos & irlandés & católico & soltero & mozo de Donnoghe \\
\hline \multirow{3}{*}{ cuartel 13} & Samuel Hondubro & ingles & protestante & $\mathrm{s} / \mathrm{d}$ & oficial zapatero \\
\hline & Diego Gordon & ingles & protestante & soltero & piloto \\
\hline & Pedro Ruirdoni & irlandés & católico & $\mathrm{s} / \mathrm{d}$ & sastre \\
\hline \multirow[t]{6}{*}{ cuartel 14} & J uan Tindall & ingles & católico rec & $\mathrm{s} / \mathrm{d}$ & prof de medicina \\
\hline & J uan Guarguens & ingles & protestante & soltero & botero \\
\hline & Lucia Hutet & ingles & $\mathrm{s} / \mathrm{d}$ & soltero & $\mathrm{s} / \mathrm{d}$ \\
\hline & $\begin{array}{|lc|}\text { ingleses } 15 & 53,57 \% \\
\text { irlandeses } 10 & 35,71 \% \\
\text { escoceses } 2 & 7,14 \%\end{array}$ & & $\begin{array}{l}\text { católicos } \\
\text { protestantes } \\
\text { s/d } \\
\end{array}$ & $\begin{array}{r}15 \\
5 \\
8 \\
\end{array}$ & $\begin{array}{l}53,57 \% \\
17,86 \% \\
28,57 \% \\
\end{array}$ \\
\hline & s/d $1 \quad 3,57 \%$ & & total & 28 & $100 \%$ \\
\hline & total 28 & & & & \\
\hline
\end{tabular}


Cuadro 7- Empadronamiento años 1806/ 7

\begin{tabular}{|l|l|l|l|}
\hline \multirow{4}{*}{ Cuartel } & \multicolumn{3}{l|}{ EMPADRONAMIENTO AÑOS 1806/7 } \\
\hline & mujeres & total & \% \\
\hline cuartel 1 & 340 & 615 & 55,28 \\
\hline cuartel 2 & 1219 & 2166 & 56,28 \\
\hline cuartel 3 & s/d & & \\
\hline cuartel 5 & 2105 & 4331 & 48,6 \\
\hline cuartel 6 & 1475 & 2841 & 51,92 \\
\hline cuartel 7 & s/d & & \\
\hline cuartel 9 & s/d & & \\
\hline cuartel 11 & s/d & & \\
\hline cuartel 13 & s/d & & \\
\hline cuartel 17 & s/d & & \\
\hline & 5139 & $\mathbf{9 9 5 3}$ & $\mathbf{5 1 , 6 3}$ \\
\hline
\end{tabular}

Cuadro 8- Empadronamiento año 1810

\begin{tabular}{|l|c|c|c|}
\hline \multirow{2}{*}{ Cuadro 8 } & \multicolumn{3}{l|}{ EMPADRONAMIENTO AÑO 1810 } \\
\cline { 2 - 4 } & mujeres & total & \% \\
\hline cuartel 2 & 1241 & 2280 & 54,43 \\
\hline cuartel 4 & 752 & 2242 & 33,54 \\
\hline cuartel 5 & 1890 & 4237 & 44,61 \\
\hline cuartel 6 & 1248 & 2519 & 49,54 \\
\hline cuartel 8 & 1423 & 3302 & 43,1 \\
\hline cuartel 10 & 402 & 976 & 41,19 \\
\hline cuartel 11 & $\mathrm{s} / \mathrm{d}$ & & \\
\hline cuartel 12 & 1012 & 2033 & 49,78 \\
\hline cuartel 13 & 1260 & 2738 & 46,02 \\
\hline cuartel 14 & 1311 & 2517 & 52,09 \\
\hline cuartel 15 & 873 & 1930 & 45,23 \\
\hline cuartel 17 & 1043 & 2064 & 50,53 \\
\hline cuartel 18 & 875 & 1861 & 47,02 \\
\hline cuartel 20 & s/d & & \\
\hline & $\mathbf{1 3 3 3 0}$ & $\mathbf{2 8 6 9 9}$ & $\mathbf{4 6 , 4 5}$ \\
\hline
\end{tabular}


Cuadro 9- Empadronamiento años 1806/ 1807

\begin{tabular}{|c|c|c|c|c|c|c|c|c|c|c|c|c|c|c|c|c|c|c|c|}
\hline \multirow[b]{2}{*}{ Cuartel } & \multirow[b]{2}{*}{ criollos } & \multirow[b]{2}{*}{$\%$} & \multirow[b]{2}{*}{ Españ/ eur* } & \multirow[b]{2}{*}{$\%$} & \multirow[b]{2}{*}{ Indios } & \multirow[b]{2}{*}{$\%$} & \multicolumn{4}{|c|}{$\begin{array}{l}\text { EMPADRONAMIENTO AÑOS } \\
1806 / 7\end{array}$} & \multirow[b]{2}{*}{ extranj* } & \multirow[b]{2}{*}{$\%$} & \multirow[b]{2}{*}{ brit+ } & \multirow[b]{2}{*}{$\%$} & \multirow[b]{2}{*}{ s/clasific } & \multirow[b]{2}{*}{$\%$} & \multirow[b]{2}{*}{ religiosos } & \multirow[b]{2}{*}{$\%$} & \multirow[b]{2}{*}{ total } \\
\hline & & & & & & & pard/neg/mul & $\%$ & Esclavos & $\%$ & & & & & & & & & \\
\hline cuartel 1 & 383 & & 37 & & & & 45 & & 57 & & 4 & & & & 36 & & 57 & & 619 \\
\hline cuartel 2 & & & 1289 & & 52 & & 296 & & 498 & & 18 & & & & & & & & 2153 \\
\hline cuartel 3 & 469 & & 134 & & 6 & & & & 468 & & 30 & & & & 253 & & 67 & & 1427 \\
\hline cuartel 4 & & & & & & & & & & & 25 & & 1 & & & & & & 25 \\
\hline cuartel 5 & & & 3166 & & 81 & & 204 & & 950 & & 49 & & 2 & & & & & & 4450 \\
\hline cuartel 6 & & & 1959 & & 141 & & 741 & & & & 37 & & 1 & & & & & & 2878 \\
\hline cuartel 7 & & & & & & & & & & & 57 & & & & & & & & 57 \\
\hline cuartel 8 & & & & & & & & & & & 34 & & 1 & & & & & & 34 \\
\hline cuartel 9 & & & 1808 & & 5 & & & & 606 & & 23 & & 1 & & & & & & 2442 \\
\hline cuartel 10 & & & & & & & & & & & 3 & & 1 & & & & & & 3 \\
\hline cuartel 11 & 485 & & 47 & & 22 & & 0 & & 35 & & & & & & 457 & & & & 1046 \\
\hline cuartel 12 & & & & & & & & & & & 6 & & & & & & & & 6 \\
\hline cuartel 13 & 180 & & 262 & & 3 & & & & 822 & & 35 & & & & 1536 & & & & 2838 \\
\hline cuartel $14 \mathrm{x}$ & & & & & & & & & & & 23 & & 1 & & & & & & 23 \\
\hline cuartel 15 & & & & & & & & & & & 15 & & & & & & & & 15 \\
\hline cuartel 16 & & & & & & & & & & & 9 & & & & & & & & 9 \\
\hline cuartel 17 & 464 & & 101 & & 6 & & & & 245 & & 11 & & & & 1047 & & & & 1874 \\
\hline cuartel 18 & & & & & & & & & & & 10 & & 1 & & & & & & 10 \\
\hline cuartel 19 & 984 & & 100 & & 31 & & 133 & & 148 & & 6 & & & & & & & & 1402 \\
\hline cuartel 20 & & & & & & & & & & & 2 & & 1 & & & & & & 2 \\
\hline & 2965 & 13,91 & 8903 & 41,77 & 347 & 1,63 & 1419 & 6,66 & 3829 & 18 & 397 & 1,86 & 10 & 0,05 & 3329 & 15,62 & 124 & 0,58 & 21313 \\
\hline
\end{tabular}

(*) Según el empadronamiento de extranjeros (número superior a los resúmenes de los padrones)

(+) incluimos a los irlandeses

(x) El británico figura como J ayme Erzano, irlandés, herrero, soltero, 58 años (en el resumen figura J ayme Egans, de Dublin) 
Cuadro 10 - Empadronamiento año 1810

\begin{tabular}{|c|c|c|c|c|c|c|c|c|c|c|c|c|c|c|c|c|c|c|c|}
\hline \multirow[b]{2}{*}{ Cuartel } & \multirow[b]{2}{*}{\begin{tabular}{|l|} 
criollos/ am \\
er
\end{tabular}} & \multirow[b]{2}{*}{$\%$} & \multirow[b]{2}{*}{ Españ/ eur } & \multirow[b]{2}{*}{$\%$} & \multirow[b]{2}{*}{ Indios } & \multicolumn{4}{|c|}{$\begin{array}{l}\text { EMPADRONAMIENTO } \\
\text { AÑO } 1810\end{array}$} & \multirow[b]{2}{*}{$\%$} & \multirow[b]{2}{*}{ brit } & \multirow[b]{2}{*}{$\%$} & \multirow[b]{2}{*}{$\begin{array}{l}\text { extranjer } \\
\text { os }\end{array}$} & \multirow[b]{2}{*}{$\%$} & \multirow[b]{2}{*}{ sin clasific* } & \multirow[b]{2}{*}{$\%$} & \multirow[b]{2}{*}{ relig/ presb } & \multirow[b]{2}{*}{$\%$} & \multirow[b]{2}{*}{ total } \\
\hline & & & & & & $\%$ & $\begin{array}{l}\text { pard/neg/ } \\
\text { mul }\end{array}$ & $\%$ & Esclavos & & & & & & & & & & \\
\hline cuartel 2 & 1175 & & 133 & & 20 & & 335 & & 579 & & & & 38 & & & & & & 2280 \\
\hline cuartel 4 & 939 & & 345 & & & & & & 912 & & & & 46 & & & & & & 2242 \\
\hline cuartel 5 & 2785 & & 359 & & & & & & 1005 & & & & 88 & & & & & & 4237 \\
\hline cuartel 6 & 1647 & & 178 & & & & & & 668 & & & & 26 & & & & & & 2519 \\
\hline cuartel 8 & 1377 & & 448 & & & & & & & & & & 92 & & 1385 & & & & 3302 \\
\hline cuartel 10 & 639 & & 63 & & 9 & & 119 & & 132 & & & & 14 & & & & & & 976 \\
\hline Cuartel 11 & 1045 & & 61 & & 35 & & 44 & & 88 & & & & 10 & & 30 & & & & 1313 \\
\hline cuartel 12 & 373 & & 116 & & 10 & & 130 & & 479 & & & & 9 & & 909 & & 7 & & 2033 \\
\hline cuartel 13 & 784 & & 225 & & & & & & 1094 & & & & 40 & & 595 & & & & 2738 \\
\hline cuartel 14 & 805 & & 167 & & & & & & 833 & & & & 47 & & 665 & & & & 2517 \\
\hline cuartel 15 & 863 & & 65 & & & & & & 495 & & & & 31 & & 476 & & & & 1930 \\
\hline cuartel 17 & 791 & & 104 & & & & 305 & & 340 & & & & 13 & & 511 & & & & 2064 \\
\hline cuartel 18 & 764 & & 119 & & & & 98 & & 348 & & & & 18 & & 514 & & & & 1861 \\
\hline cuartel 20 & 654 & & 35 & & 73 & & 89 & & 1114 & & & & & & & & & & 1965 \\
\hline & 14641 & 45,79 & 2418 & 7,56 & 147 & 0,46 & 1120 & 3,5 & 8087 & 25,3 & & 0 & 472 & 1,48 & 5085 & 15,9 & 7 & 0,02 & 31977 \\
\hline
\end{tabular}

Consideramos que los indios, pardos, mulatos y negros libres fueron clasificados como americanos

Los niños de esclavos fueron sumados como esclavos, el resto de niños y niñas fueron considerados americanos.

(*) Mujeres españolas, americanas y personas sin nacionalidad determinada 


\section{Referências}

ALZAGA, Enrique W. Fuga del General Beresford, 1807. Buenos Aires: Emecé, 1965.

BATTOLLA, Octavio. Los primeros ingleses en Buenos Aires 1780-1830. Buenos Aires: Ed. Muro, 1928.

COGHLAN, Eduardo. Los irlandeses en la Argentina. Su situación y descendencia. Buenos Aires: Abraxas, 1987.

FITTE, Ernesto. Crónica de un cónsul oficioso británico. Buenos Aires: A.N.H., 1964.

FITTE, Ernesto. Los comerciantes ingleses en las vísperas de la revolución de Mayo. Buenos Aires: A.N.H., 1967.

GARCÍA BELSUNCE, César (Dir.). Buenos Aires. Su gente (1800-1830). Buenos Aires: Compañía impresora argentina, 1976. t. 1.

GILLESPIE, Alexander. Buenos Aires y el interior. Observaciones reunidas durante una larga residencia, 1806-1807. Buenos Aires: A-Z Editora, 1994.

GRAHAM-YOOLL, Andrew. Pequeñas guerras británicas en América latina. Buenos Aires: Ed. Legasa, 1987.

GRAHAM-YOOLL, Andrew. La colonia olvidada. Buenos Aires: Ed. Sudamericana, 2000.

HANON, Maxine. Diccionario de Británicos en Buenos Aires. Buenos Aires: Gutten Press, 2005.

NÚCLEO ARGENTINO DE ESTUDIOS HISTÓRICOS. Británicos vistos por ojos argentinos. Buenos Aires: Editorial Multi Revista, 1941.

PELLEGRINI, Carlos. Los británicos en la Argentina. Conferencia .pronunciada por el Dr. Carlos Pellegrini en el Prince George's may. Buenos Aires, 1905.

ROBERTS, Carlos. Las invasiones inglesas del Río de la Plata. Buenos Aires: Emece, 2006.

STREET, J ohn. Gran Bretaña y la- independencia del Río de la Plata. Buenos Aires: Paidos, 1967.

TODO es Historia. Buenos Aires, n. 468, jul. 2006.

\section{Fuentes Documentales}

EMPADRONAMIENTOS de los años 1804, 1806/7 y 1810 Tomados de la obra dirigida por:

RAVIGNANI, Emilio, Documentos para la historia argentina. Buenos Aires: Facultad de Filosofía y Letras, Universidad de Buenos Aires, Compañía Sudamericana de Billetes de Banco, 1919. t. 12. 
CARTAS y documentos de 1806, 1809 y 1810 Tomados de:

FITTE, Ernesto. Los comerciantes ingleses en las vísperas de la revolución de Mayo. Buenos Aires: A.N.H., 1967.

RAVIGNANI, Emilio. Documentos para la historia argentina. Buenos Aires: Facultad de Filosofía y Letras, Universidad de Buenos Aires, Compañía Sudamericana de Billetes de Banco, 1919. t. 9.

RAVIGNANI, Emilio. Mayo Documental. Buenos Aires: Instituto de Historia Argentina, 1962. t.9.

Colaboração recebida em 28/ 12/ 2009 e aprovada em 21/ 04/ 2010. 